\title{
Transition from the Seniority to the Anharmonic Vibrator Regime in Nuclei
}

\author{
R. Bijker ${ }^{1}$, A. Frank ${ }^{1,2}$ and S. Pittel ${ }^{3}$ \\ ${ }^{1}$ Instituto de Ciencias Nucleares, U.N.A.M., A.P. 70-543, 04510 México, D. F., México \\ ${ }^{2}$ Instituto de Fúsica, Laboratorio de Cuernavaca, U.N.A.M., A.P. 139-B, Cuernavaca, Morelos, \\ México \\ ${ }^{3}$ Bartol Research Institute, University of Delaware, Newark, DE 19716, U.S.A.
}

\begin{abstract}
A recent analysis of experimental energy systematics suggests that all collective nuclei fall into one of three classes - seniority, anharmonic vibrational, or rotational - with sharp phase transitions between them. We investigate the transition from the seniority to the anharmonic vibrator regime within a shell model framework involving a single large $j$-orbit. The calculations qualitatively reproduce the observed transitional behavior, both for $U(5)$ like and $O(6)$ like nuclei. They also confirm the preeminent role played by the neutron-proton interaction in producing the phase transition.
\end{abstract}

PACS numbers: 21.10.-k, 21.60.-n 
A recent analysis of the energy systematics of the lowest $2^{+}$and $4^{+}$states in collective even-even nuclei has shown some remarkable features [1,2]. These data suggest that medium-weight and heavy collective even-even nuclei fall into three classes: (a) nuclei dominated by a generalized-seniority structure, (b) anharmonic vibrational nuclei, and (c) welldeformed rotational nuclei. In each of these three regimes, the energy systematics is extremely robust, suggesting an underlying simplicity of low-energy nuclear structure never before appreciated. Furthermore, the transitions between regimes occur very rapidly, typically with the addition or removal of only one or two pairs of nucleons. Since these observations were reported, much work has been carried out to understand their significance. There is now a fairly good - albeit general - understanding of the three regimes themselves [2] and of the transition from anharmonic vibrational to rotational nuclei.

In this work we study the transition from the seniority to the anharmonic vibrator regime. In the seniority region, the energies of the lowest $4^{+}$and $2^{+}$states are related by the simple formula $E\left(4_{1}^{+}\right)=E\left(2_{1}^{+}\right)+\epsilon_{4}$, where $\epsilon_{4}$ is constant in each nuclear region. Nuclei falling into this class are typically either semi-magic or nearly semi-magic. For the anharmonic vibrator regime, on the other hand, the corresponding relation is $E\left(4_{1}^{+}\right)=2 E\left(2_{1}^{+}\right)+\epsilon_{4}$, with $\epsilon_{4} \approx 156 \mathrm{keV}$ in the entire $Z=38-82$ region and with $\epsilon_{4} \approx 67 \mathrm{keV}$ in the actinides. Nuclei that fall into this class include those characteristically thought of as vibrational (or $U(5)$ like) and gamma-soft (or $O(6)$ like).

To gain a more fundamental insight into this transition, we have carried out a series of shell-model calculations. The calculations were performed under the simplifying assumption that the neutrons and protons each occupy a single large $j=15 / 2$ orbital. The calculations were carried out for 0 to 6 protons with either 0 to 6 neutrons or 0 to 6 neutron holes, since in both cases the data show transitions to an anharmonic vibrational behavior.

Despite the assumption of a single active $j$-orbit, the dimensions increase rapidly, reaching 3199 for four active protons $(Z=4)$, four active neutrons $(N=4)$ and $J^{\pi}=4^{+}$. Increasing the angular momentum of the single $j$-orbit or increasing the number of active particles make the calculations prohibitive. As such, the calculations that we report should 
not be directly interpreted in terms of a real nuclear region, but rather as a schematic study of the mechanism underlying the transition from one regime to the other. Similarly, the hamiltonian strength parameters, described below, represent effective values.

We use a shell-model hamiltonian with the following ingredients:

- The interaction between protons is assumed to be a surface delta interaction (SDI) of strength $G_{p p}=0.24 \mathrm{MeV}$, supplemented by a quadrupole interaction of strength $\chi_{p p}=0.6 \mathrm{MeV}$ to bring down the energy of the lowest $2^{+}$state in semi-magic nuclei to a value compatible with data for such nuclei.

- The interaction between neutrons is likewise taken to be a quadrupole-enhanced SDI. When the neutrons are particle-like, we choose a strength $G_{n n}=0.22 \mathrm{MeV}$, somewhat weaker than for protons to simulate a small neutron excess. When they are hole-like, so that the excess is even larger, we assume a strength of $G_{n n}=0.20 \mathrm{MeV}$. In both cases, the strength of the quadrupole interaction is chosen to be $\chi_{n n}=1.0 \mathrm{MeV}$.

- Finally, for the neutron-proton interaction, we assume a pure quadrupole force, with strength $\chi_{n p}=10 \mathrm{MeV}$.

The quadrupole strength parameters given above include the single relevant radial matrix element.

Table [ summarizes the results we obtain for the energies of the lowest $2^{+}$and $4^{+}$states for all systems that we considered. The results clearly exhibit the systematics of the data noted earlier. Namely:

- For semi-magic nuclei, the energy of the lowest $4^{+}$state in all cases is a few hundred $\mathrm{keV}$ above that of the lowest $2^{+}$state. This is a pattern typical of spherical nuclei in the seniority regime.

- When particles are either added to or removed from the closed shell of the semi-magic nucleus, there is a rapid change in the behavior of the lowest $2^{+}$and $4^{+}$energies. 
When both the neutrons and protons are particle-like, adding just two particles to a semi-magic seniority nucleus raises the ratio $R=E\left(4_{1}^{+}\right) / E\left(2_{1}^{+}\right)$to a value greater than 2 (except when $Z=N=2$ where it only rises to 1.8). Successive addition of nucleons further raises this ratio, albeit only slightly. A similar, though not quite as strong, effect is seen for nuclei involving proton particles and neutron holes. Adding two holes to the semi-magic nucleus quickly raises $R$ to about 1.8 or 1.9 (except when $Z=2$ and $N=-2$ where it is smaller). This is precisely the pattern seen in the data; a rapid transition from the seniority regime to the anharmonic vibrator regime. Especially interesting is that (as noted above) the same behavior was found (with minor quantitative differences) in the transitions to $U(5)$ nuclei (both neutrons and protons particle-like) and $O(6)$ nuclei (one type of nucleon particle-like and the other hole-like).

The fact that our calculations, despite their simplicity, reproduce reasonably well the qualitative trends observed in the energy systematics lends hope that they may shed light on the physics underlying the phase transition. With that in mind, we now turn to a discussion of the wave functions that emerged from our calculations. We concentrate on a limited set of representative results to illustrate the key points.

In Table [I], we present results for systems with 4 active protons and differing numbers of active neutrons or neutron holes ranging from 0 to 4 . More specifically, we give the probability amplitudes for different seniority configurations $\left(v_{p}, v_{n}\right)$, where $v_{p}$ and $v_{n}$ denote the proton and neutron seniorities, respectively. (Note that since we have limited our analysis to a single $j$-orbit, these are real and not generalized seniorities [3].)

When there is only one type of nucleon $(Z=4, N=0)$, the lowest $2^{+}$and $4^{+}$states are almost pure in their seniority structure. That they are not exact seniority eigenstates is a consequence of the additional quadrupole component included in the two-proton interaction.

As neutron pairs are added or removed, the structure of the eigenstates changes dramatically. The lowest $2^{+}$and $4^{+}$states immediately develop a strongly mixed seniority 
structure, due to the seniority-breaking neutron-proton quadrupole force. The mixing is already significant with the addition of only 2 neutrons (or neutron holes) and becomes stronger with the addition of two more. Associated directly with this seniority mixing is the rapid development of an anharmonic vibrator energy pattern.

That the neutron-proton interaction is responsible for the onset of collectivity and that seniority mixing plays a central role in this phenomenon are already well known facts $\llbracket$ | There are two specific features that appear in our results, however, which we do not believe are as well known and on which we therefore wish to elaborate. The first is that precisely the same features occur when one adds neutrons or neutron holes. From the table, we see that qualitatively the same mixing shows up in both cases. Quantitatively, the mixing is somewhat weaker in the case of neutron holes, and this is directly correlated with the fact that the $4^{+}$to $2^{+}$energy ratio is not quite as large there.

The second point we would like to note concerns some important differences that evolve in the structure of the lowest $2^{+}$and $4^{+}$states when neutrons or neutron holes are added to the semi-magic system. As we can see from the table, the $4^{+}$wave functions typically have a larger component of $\left(v_{p}, v_{n}\right)=(4,0)$ than $(2,0)$. This is to be contrasted with the $2^{+}$ states, for which the $(2,0)$ configuration is always more important. What is the reason for this and what (if anything) is its impact on nuclear collectivity?

The answer to these questions arises from considering the pair structure of these seniority configurations. In the case of $2^{+}$states, the $(2,0)$ configuration involves a single $D$-pair (with $J^{\pi}=2^{+}$), while the lowest $(4,0)$ configuration involves two $D$-pairs. In the case of $4^{+}$ states, the $(2,0)$ configuration involves a single $G$-pair (with $J^{\pi}=4^{+}$) and the lowest $(4,0)$ configuration corresponds to two $D$-pairs. In the semi-magic system, the $4^{+}$configuration with a single $G$-pair is lower than that with two $D$-pairs (for any reasonable force). What our results indicate is that when particles of the other type are added to (or removed from) a semi-magic nucleus, the configuration with two $D$-pairs is effectively lowered below that of the single $G$-pair configuration. A more detailed analysis shows that this lowering takes place through off-diagonal (and not diagonal) matrix elements of the neutron-proton interaction, 
and this is the reason we use the term effectively to describe the phenomenon. This effective crossing has important consequences on the description of collective properties of nuclei. It provides the mechanism for $S-D$ pair dominance in the low-energy properties of nuclei with a large enough number of valence protons and neutrons, which is at the heart of the success of the Interacting Boson Model [5].

Summarizing, the calculations reported here qualitatively reproduce the recently noted transition in $4^{+}-2^{+}$energetics from seniority (or pre-collective) nuclei to anharmonic vibrational nuclei. Energetically, the behavior is no different when undergoing a transition to nuclei of $U(5)$ character or $O(6)$ character. In both cases, it is the neutron-proton interaction which is responsible for the transition. The calculations also shed light on how $S-D$ pair dominance emerges when there are enough active neutrons and active protons.

The authors would like to acknowledge useful conversations with Rick Casten and Franco Iachello. This work was supported in part by the National Science Foundation under grant \#s PHY-9303041, PHY-9600445 and INT-9314535, the European Community through project CI1*-CT94-0072, DGAPA UNAM through project IN105194 and CONACyT, Mexico, through project 400340-5-3401E. 


\section{REFERENCES}

[1] R. F. Casten, N. V. Zamfir and D. S. Brenner, Phys. Rev. Lett. 71 (1993) 227.

[2] N. V. Zamfir, R. F. Casten and D. S. Brenner, Phys. Rev. Lett. 72 (1994) 3480.

[3] I. Talmi, Simple models of complex nuclei, (Harwood Academic Publishers, 1993).

[4] P. Federman and S. Pittel, Phys. Rev. C20 (1979) 820.

[5] F. Iachello and A. Arima, The Interacting Boson Model, (Cambridge University Press, 1987). 


\section{TABLES}

TABLE I. Calculated energies of the lowest $2^{+}$and $4^{+}$states for all nuclei considered in this study. $Z$ denotes the number of active protons and $N$ the number of active neutrons (if positive) or neutron holes (if negative). $R$ denotes the ratio $R=E\left(4_{1}^{+}\right) / E\left(2_{1}^{+}\right)$. All energies are given in $\mathrm{MeV}$.

\begin{tabular}{|c|c|c|c|c|c|c|c|c|c|}
\hline$Z$ & $N$ & $E_{2_{1}^{+}}$ & $E_{4_{1}^{+}}$ & $R$ & $Z$ & $N$ & $E_{2_{1}^{+}}$ & $E_{4_{1}^{+}}$ & $R$ \\
\hline 0 & 2 & 1.35 & 1.61 & 1.19 & 0 & -2 & 1.23 & 1.47 & 1.19 \\
\hline 0 & 4 & 1.27 & 1.70 & 1.34 & 0 & -4 & 1.15 & 1.56 & 1.36 \\
\hline 0 & 6 & 1.23 & 1.76 & 1.42 & 0 & -6 & 1.11 & 1.62 & 1.45 \\
\hline 2 & 0 & 1.46 & 1.71 & 1.17 & 2 & -0 & 1.46 & 1.71 & 1.17 \\
\hline 2 & 2 & 0.56 & 1.01 & 1.79 & 2 & -2 & 0.83 & 1.23 & 1.49 \\
\hline 2 & 4 & 0.38 & 0.82 & 2.17 & 2 & -4 & 0.64 & 1.11 & 1.74 \\
\hline 2 & 6 & 0.35 & 0.79 & 2.24 & 2 & -6 & 0.49 & 0.94 & 1.93 \\
\hline 4 & 0 & 1.41 & 1.77 & 1.25 & 4 & -0 & 1.41 & 1.77 & 1.25 \\
\hline 4 & 2 & 0.40 & 0.85 & 2.14 & 4 & -2 & 0.65 & 1.11 & 1.72 \\
\hline 4 & 4 & 0.23 & 0.59 & 2.51 & 4 & -4 & 0.48 & 0.92 & 1.93 \\
\hline 6 & 0 & 1.39 & 1.80 & 1.30 & 6 & -0 & 1.39 & 1.80 & 1.30 \\
\hline 6 & 2 & 0.38 & 0.84 & 2.21 & 6 & -2 & 0.51 & 0.98 & 1.94 \\
\hline
\end{tabular}


TABLE II. Probability amplitudes of seniority configurations $\left(v_{p}, v_{n}\right)$ for the lowest $2^{+}$and $4^{+}$ states obtained in the calculations described in the text.

\begin{tabular}{|c|c|c|c|c|c|c|c|c|c|c|}
\hline \multirow[t]{2}{*}{$Z$} & \multirow[t]{2}{*}{$N$} & \multirow[t]{2}{*}{$J^{\pi}$} & \multicolumn{8}{|c|}{$\left(v_{p}, v_{n}\right)$} \\
\hline & & & $(2,0)$ & $(4,0)$ & $(0,2)$ & $(0,4)$ & $(2,2)$ & $(2,4)$ & $(4,2)$ & $(4,4)$ \\
\hline \multirow[t]{2}{*}{4} & 0 & $2^{+}$ & 0.99 & 0.01 & - & - & - & - & - & - \\
\hline & & $4^{+}$ & 0.99 & 0.01 & - & - & - & - & - & - \\
\hline \multirow[t]{2}{*}{4} & 2 & $2^{+}$ & 0.23 & 0.04 & 0.21 & - & 0.25 & - & 0.27 & - \\
\hline & & $4^{+}$ & 0.03 & 0.15 & 0.14 & - & 0.35 & - & 0.33 & - \\
\hline \multirow[t]{2}{*}{4} & 4 & $2^{+}$ & 0.12 & 0.03 & 0.12 & 0.03 & 0.12 & 0.18 & 0.17 & 0.23 \\
\hline & & $4^{+}$ & 0.01 & 0.08 & 0.01 & 0.09 & 0.15 & 0.20 & 0.18 & 0.28 \\
\hline \multirow[t]{2}{*}{4} & -2 & $2^{+}$ & 0.28 & 0.01 & 0.41 & - & 0.20 & - & 0.11 & - \\
\hline & & $4^{+}$ & 0.08 & 0.06 & 0.37 & - & 0.37 & - & 0.12 & - \\
\hline \multirow[t]{2}{*}{4} & -4 & $2^{+}$ & 0.22 & 0.01 & 0.29 & 0.02 & 0.08 & 0.18 & 0.13 & 0.08 \\
\hline & & $4^{+}$ & 0.05 & 0.08 & 0.06 & 0.16 & 0.23 & 0.17 & 0.11 & 0.14 \\
\hline
\end{tabular}

